\title{
Anaplastic ganglioglioma becoming symptomatic in the third trimester of pregnancy
}

\author{
Stylianos Pikis $^{1 *}$, Tigran Petrosyan ${ }^{1}$, Kalliopi Diamantopoulou ${ }^{2}$, Christos Kelesis $^{1}$
}

\begin{abstract}
${ }^{1}$ Department of Neurosurgery, Korgialenio Benakio Red Cross Hospital of Athens, Athens, Greece
${ }^{2}$ Department of Pathology, Korgialenio Benakio Red Cross Hospital of Athens, Athens, Greece
\end{abstract}

Received: 08 January 2017

Accepted: 08 February 2017

\section{*Correspondence:}

Dr. Stylianos Pikis,

E-mail: steliospikis@ hotmail.com

Copyright: $\odot$ the author(s), publisher and licensee Medip Academy. This is an open-access article distributed under the terms of the Creative Commons Attribution Non-Commercial License, which permits unrestricted non-commercial

\begin{abstract}
Intracranial tumors are rare in pregnancy and may present a diagnostic and therapeutic challenge. We report on a 34year-old female who presented with new-onset partial seizures hours following delivery due to a left frontal space occupying lesion. Urgent surgical resection of the lesion was performed and histopathologic evaluation revealed anaplastic ganglioglioma. Post-operatively the patient received radiotherapy and temozolamide chemotherapy. She reported a three-month history of progressive headache and nausea which were falsely attributed to pregnancy both by the patient and her treating physician leading to a delay in diagnosis and management. In the pregnant patient presenting with neurological symptoms a high index of suspicion and a thorough physical examination is required to identify those patients at risk of intracranial pathology.
\end{abstract}

Keywords: Anaplastic ganglioglioma, Brain tumor, Diagnosis, Pregnancy

\section{INTRODUCTION}

Gangliogliomas account for $1.2 \%$ to $7.6 \%$ of all primary central nervous system tumors ${ }^{1}$ with $1 \%-5 \%$ of these represented by the World Health Organization (WHO) grade III or anaplastic ganglioglioma variant. ${ }^{2}$ They are more common in older children and young adults and demonstrate a slight male predominance. ${ }^{1,2}$ Anaplastic gangliogliomas are usually located in the temporal lobe and most commonly present with seizures. ${ }^{2}$ Accelerated growth of intracranial World Health Organization (WHO) grade I and Grade II gangliogliomas during pregnancy, has been reported. ${ }^{1,3}$ We report on a rare case of a 34year-old woman presenting with new-onset partial seizures occurring within hours postpartum due to a previously unrecognized WHO grade III anaplastic ganglioglioma at the left frontal lobe.

\section{CASE REPORT}

A 34-year-old woman suffered a new-onset partial seizure hours after giving birth. Urgent brain magnetic resonance (MR) image was significant for a left frontal space occupying lesion (Figure 1). She was subsequently referred to the neurosurgery clinic for further management.

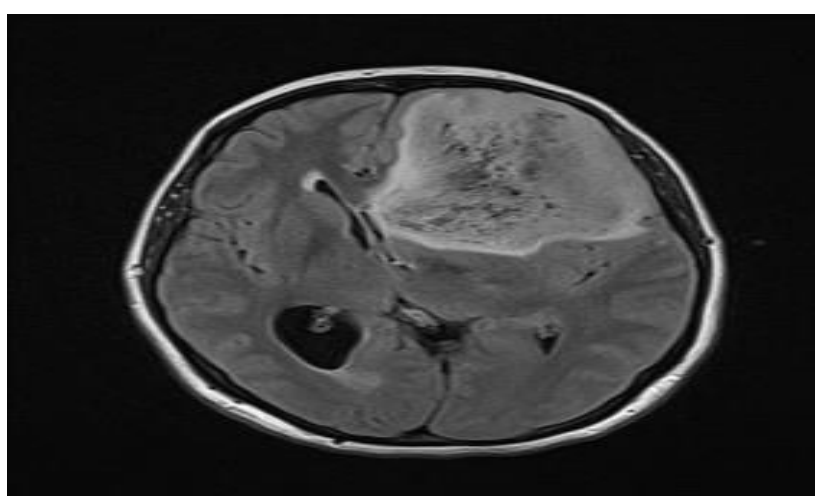

Figure 1: Axial magnetic resonance fluid attenuated inversion recovery (FLAIR) image significant for a left frontal space occupying lesion associated with midline shift. 
The patient's past medical history and admission neurological examination was unremarkable. On direct questioning, she reported a three-month history of progressive headaches and nausea she attributed to pregnancy. At surgery, partial resection of the tumor was achieved via a left frontal craniotomy.

On histopathologic evaluation, the neoplasm displayed mixed neuronal and glial elements, with focal, mildly increased mitotic activity (up to $2 \mathrm{MF} / 10 \mathrm{HPF}$ ). On immunohistochemistry, the neoplasm expressed variable immunopositivity for CD31, CD34, S-100, p53, Synaptophysin and Neurofilament. There was focal immunopositivity for Glial fibrillary acidic protein and EGFR. Fluorescence in-situ hybridization test did not reveal a deletion mutation of chromosomes $1 \mathrm{p} / 19 \mathrm{q}$ in the neoplastic cells. The above features were consistent with the diagnosis of anaplastic ganglioglioma World Health Organization Grade III.

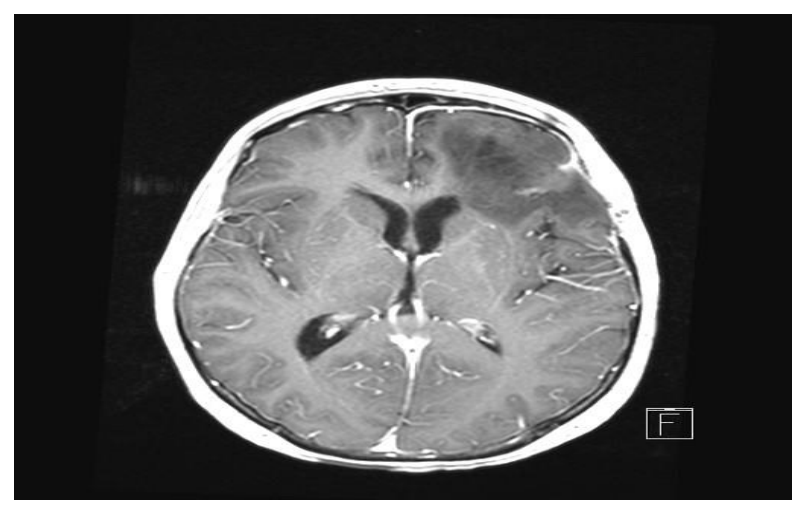

Figure 2: Post-operative, post-radiotherapy one year follow-up, axial, brain magnetic resonance $\mathrm{T} 2$ weighted image demonstrating no residual tumor enlargement.

During her hospitalization the patient remained seizure free on levetiracetam 1 gram bid. On postoperative day 14 she was discharged home with instructions to continue her anti-epileptic medication and for further oncologic management. The patient received adjuvant radiotherapy and temozolamide chemotherapy.

However, temozolamide had to be discontinued due to severe thrombocytopenia. At her one-year clinical follow-up she remained seizure free on levetiracetam 1 gram bid with no new neurologic deficit noted. Followup MR Image demonstrated no enlargement of the residual tumor (Figure 2).

\section{DISCUSSION}

Pregnancy has been associated with accelerated growth of intracranial tumors. However, the primary mechanism responsible for tumor growth during pregnancy is unclear and may involve immune tolerance, steroid-induced tumor growth, and increased mass-effect due to hemodynamic changes. ${ }^{1}$ Intracranial gangliogliomas becoming symptomatic in pregnancy or in the immediate post-partum period have been rarely reported. ${ }^{1,3}$

Knafo et al, reported on a 32-year old pregnant woman with a known WHO Grade I ganglioglioma which demonstrated increased growth rate on image during pregnancy leading to clinical deterioration and requiring surgical resection of the lesion three months after delivery. Ravindra et al, reported on a young female in the $26^{\text {th }}$ week of gestation presenting with obstructive hydrocephalus due to a WHO Grade II ganglioglioma in the pineal region requiring urgent endoscopic third ventriculostomy.

Post-partum due to continued enlargement of the tumor the patient underwent surgical resection of the tumor and adjuvant radiotherapy only to succumb to her disease two years after initial presentation. ${ }^{1}$ Our patient experienced progressive headaches and nausea during the third trimester of her pregnancy which she falsely attributed to gestation. Hours following delivery she suffered from partial seizures and following brain MR image she was diagnosed with a left frontal space occupying lesion. Surgical resection and histologic evaluation of the tumor revealed anaplastic ganglioglioma.

Intracranial tumors diagnosed during a viable pregnancy are rare and they present a complex challenge for the neurosurgeon and the obstetrician managing the patient. Decisions regarding the ideal timing for neurosurgical intervention and delivery will depend on the severity of neurological symptoms, the maternal mortality and morbidity risk, the gestational age of the embryo, and the presumed tumor histology.

Involvement of a multidisciplinary team, including neurosurgeons, obstetrician, anesthesiologist, intensive care specialist, and potentially an oncologist is necessary, with the pregnant woman's full participation in decisions. ${ }^{4}$

\section{CONCLUSION}

In conclusion, diagnosis of a supratentorial anaplastic ganglioglioma in pregnancy is rare. Non-specific symptoms of headache, nausea, and vomiting associated with intracranial tumors may be falsely attributed to pregnancy leading to a delay in diagnosis.

Thus, in the pregnant patient presenting with non-specific symptoms a high index of suspicion is required and a thorough history and physical examination should be performed to identify those patients at risk for intracranial tumor.

\author{
Funding: No funding sources \\ Conflict of interest: None declared \\ Ethical approval: Not required
}




\section{REFERENCES}

1. Ravindra VM, Braca III JA, Jensen RL, Duckworth EA. Management of intracranial pathology during pregnancy: Case example and review of management strategies. Surg Neurol Int. 2015;6:43.

2. Lucas Jr JT, Huang AJ, Mott RT, Lesser GJ, Tatter SB, Chan MD. Anaplastic ganglioglioma: a report of three cases and review of the literature. J Neurooncol 2015;123(1):171-7.

3. Knafo S, Goutagny S, Pallud J. Increased growth rate of a WHO grade I ganglioglioma during pregnancy. British J Neurosurg. 2013;27(1):119-121.
4. Pikis S, Cohen JE, Rosenthal G, Barzilay Y, Kaplan L, Shoshan Y, et al. Spinal meningioma becoming symptomatic in the third trimester of pregnancy. J Clin Neurosci. 2013;20(12):1797-9.

Cite this article as: Pikis S, Petrosyan T, Diamantopoulou K, Kelesis C. Anaplastic ganglioglioma becoming symptomatic in the third trimester of pregnancy. Int J Reprod Contracept Obstet Gynecol 2017;6:1158-60. 